\title{
Chronic Synovitis of Wrist: A Diagnostic Dilemma \& Role of Arthroscopy
}

\author{
Jawed Akram*, Gupta Vikas, Kotwal P, Gupta Rajiva, Jain Prathmesh \\ Department of Orthopaedics (AIIMS), Max Institute of Musculo-skeletal Sciences (MIMS), India
}

*Corresponding author: Jawed Akram, Department of Orthopaedics (AIIMS),

Max Institute of Musculo-skeletal Sciences (MIMS), India
Received Date: May 17, 2021

Published Date: October 05, 2021

\section{Abstract \\ Chronic Synovitis presents a diagnostic dilemma for clinicians and surgeons. In our study we investigated patients presenting with chronic synovitis of wrist joint with wrist arthroscopy. 8 out of 34 (23.53\%) of cases were histologically positive for granulomas suggestive of tuberculosis. We were able to predict the etiology of the disease based on the arthroscopy findings.}

\section{Introduction}

Chronic synovitis of wrist joint is a common manifestation seen in a majority of cases of inflammatory arthritis especially rheumatoid arthritis, the involvement in these cases is usually poly-articular, but occasionally oligo- articular and mono articular involvement may be seen. In the Indian population tuberculosis of wrist joint is an important differential diagnosis to be kept when a patient presents with Mon-articular wrist swelling.

\section{Methods}

We investigated 34 patients presenting to our clinic with symptoms of chronic synovitis of wrist joint in the time duration between January 2014 to June 2021. We included the patients with a single joint involvement excluding those with oligo articular or poly articular involvement. All of these patients have been treated initially with non-steroidal anti-inflammatory drugs. We excluded patients with symptoms less than three months duration. The patients were investigated and scheduled for diagnostic arthroscopy with synovial biopsy. None of the patients complained of fever as the presenting complaint. Family history positive for tuberculosis was present in two of the patients. Chest X-rays were normal in all but one patient which showed right upper lobe infiltrates [Table 1]. The mean ESR was recorded to be 38.7 [Table 2]. All of the patients had a past history of BCG vaccination. Past history of tuberculosis was present in one patient ten years back which was treated with directly observed treatment for a period of six months. The rheumatoid factor was positive in eleven of the cases. The mean duration of involvement in these cases was 5.5 months. X-rays of wrist joint were taken and found to be normal in 22 out of 24 cases whereas the rest 12 there were features like juxta-articular osteopenia, periarticular erosion etc. Wrist arthroscopy was performed in these patients with a diagnostic intent and synovial biopsy was taken from the involved synovium which was sent for histopathological examination.

Table 1: clinical detail.

\begin{tabular}{|c|c|c|}
\hline Histopathology & Granulomatous & Non granulomatous \\
\hline Cases & 8 & 0 \\
\hline Family h/o tuberculosis & 4 & 0 \\
\hline Chest Infilterates & 2 & 40.92 \\
\hline Mean ESR & 34 & 8 \\
\hline Abnormal radiology & 4 & 8 \\
\hline
\end{tabular}


Table 2: Arthroscopic findings in the 2 groups.

\begin{tabular}{|c|c|c|}
\hline Histopathology & Granulomatous Chr. Inflam. & Non granulomatous Chr. Inflam. \\
\hline No. of cases & 8 & 26 \\
\hline Pannus formation & 0 & 22 \\
\hline Hypertrophic synovium & 0 & 22 \\
\hline Atrophic villi & 0 & 0 \\
\hline Rice like bodies & 4 & 26 \\
\hline
\end{tabular}

\section{Literature Review}

Reports tuberculosis infections of wrist have been scarce, especially during past 30 years. Role of arthroscopy of wrist joint for tuberculosis infection has not been reported as yet. Arthroscopy has now been a standard therapy for rheumatoid arthritis of wrist allowing effective pain relief and high patient satisfaction [1]. Most of the report of tuberculosis within literature concentrate around atypical mycobacterial infection. Tuberculosis infection in wrist joint can come of variety of pathology including tuberculous arthritis, synovitis, tenosynovitis, soft tissue tuberculosis. Most striking feature of previous studies was delay between the onset of symptoms and the correct diagnosis. Most of earlier studies revealed an absence of pulmonary symptoms [2]. [Figure 1] Formation of rice like bodies has been explained in an earlier case report [3]. Earlier series express open synovectomy of wrist both as a diagnostic and therapeutic tool [4].

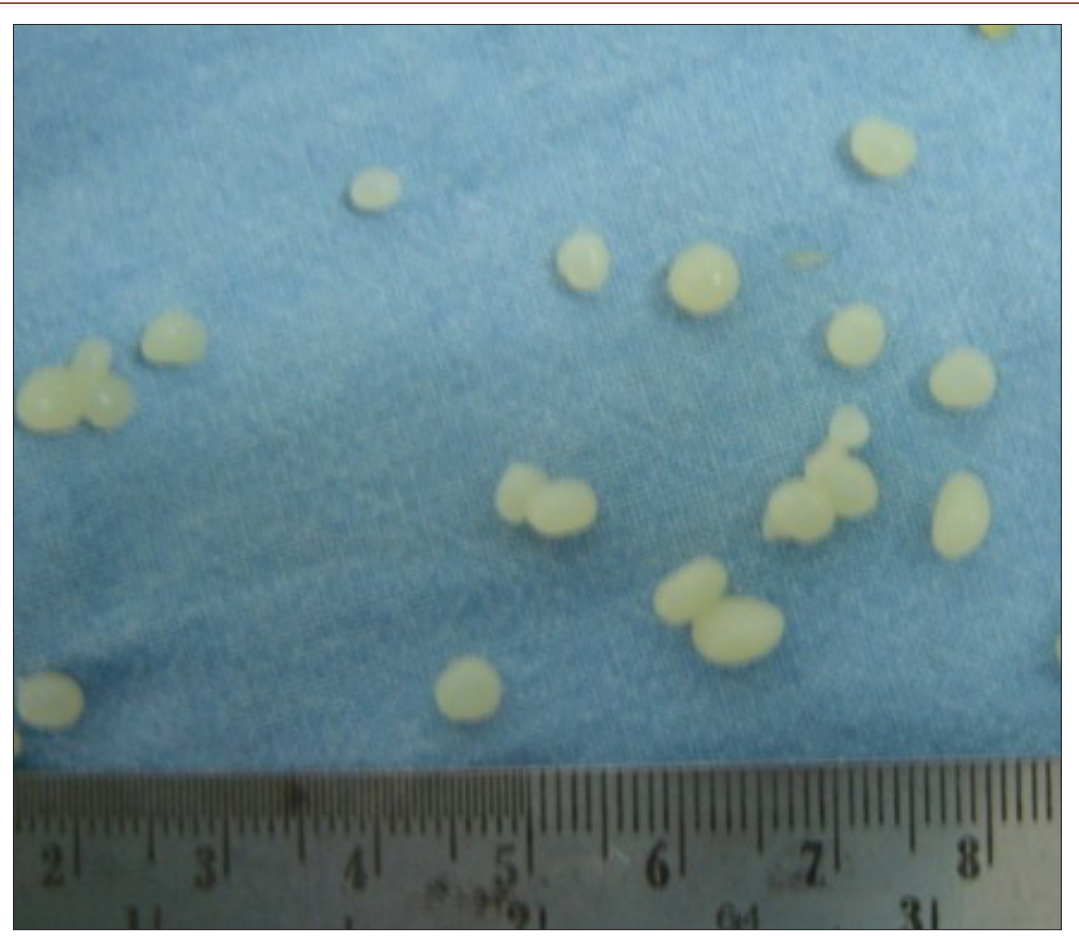

Figure 1: Rice like bodies.

Surgical technique: The 3-4 and 4-5 portals were used as standard entry portals for radio-carpal joint. Additional 1-2 and $6 \mathrm{U}$ portals were also used occasionally to view dorsal side of radiocarpal joint. Adequate synovial biopsy specimen was taken from the region of synovium which visibly seemed to be maximally involved [Figure 2]. We noticed some peculiar findings in a subgroup of patients which didn't comply with the characteristically described synovium in inflammatory diseases like rheumatoid arthritis. The peculiar features which were observed in these patients were absence of hypertrophic synovium (12 out of 34), absence of pannus formation (18 out of 34), atrophic villi (12 out of 34). 4 of the patients revealed small rice like bodies from the synovium [Figure 3]. 


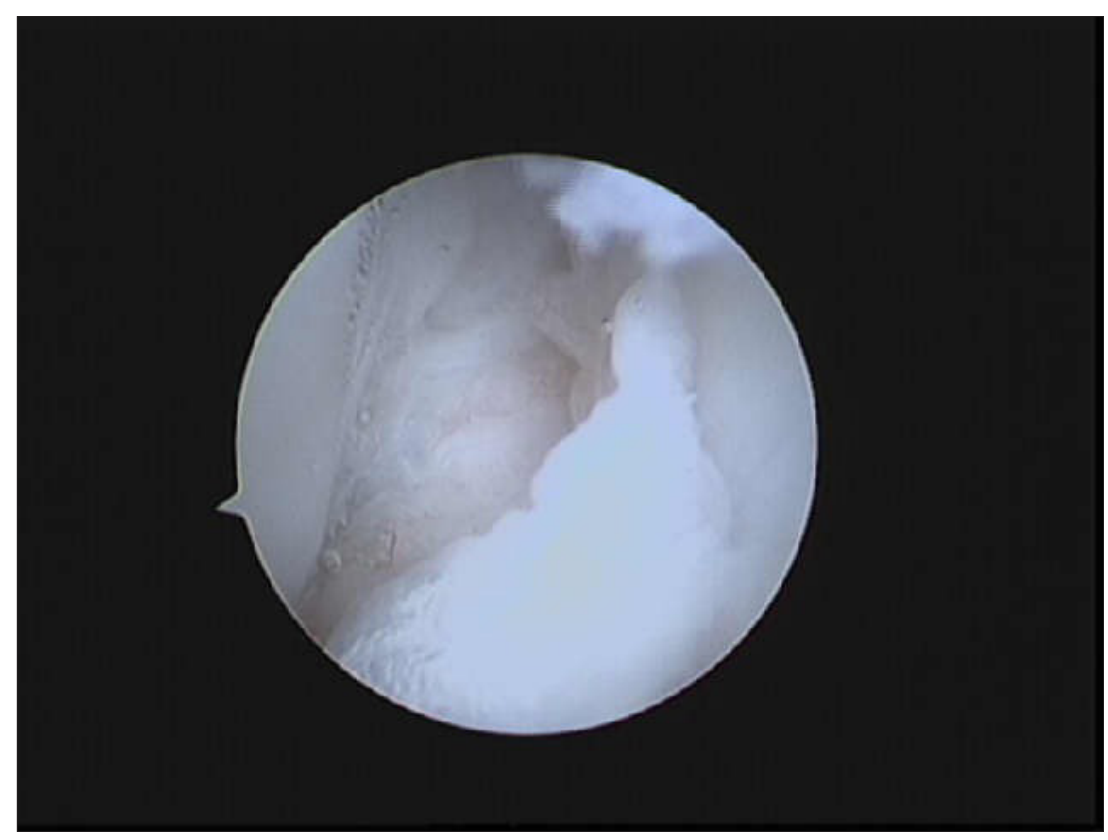

Figure 2: Synovium of a joint with histologically proven granulomatous inflammation.

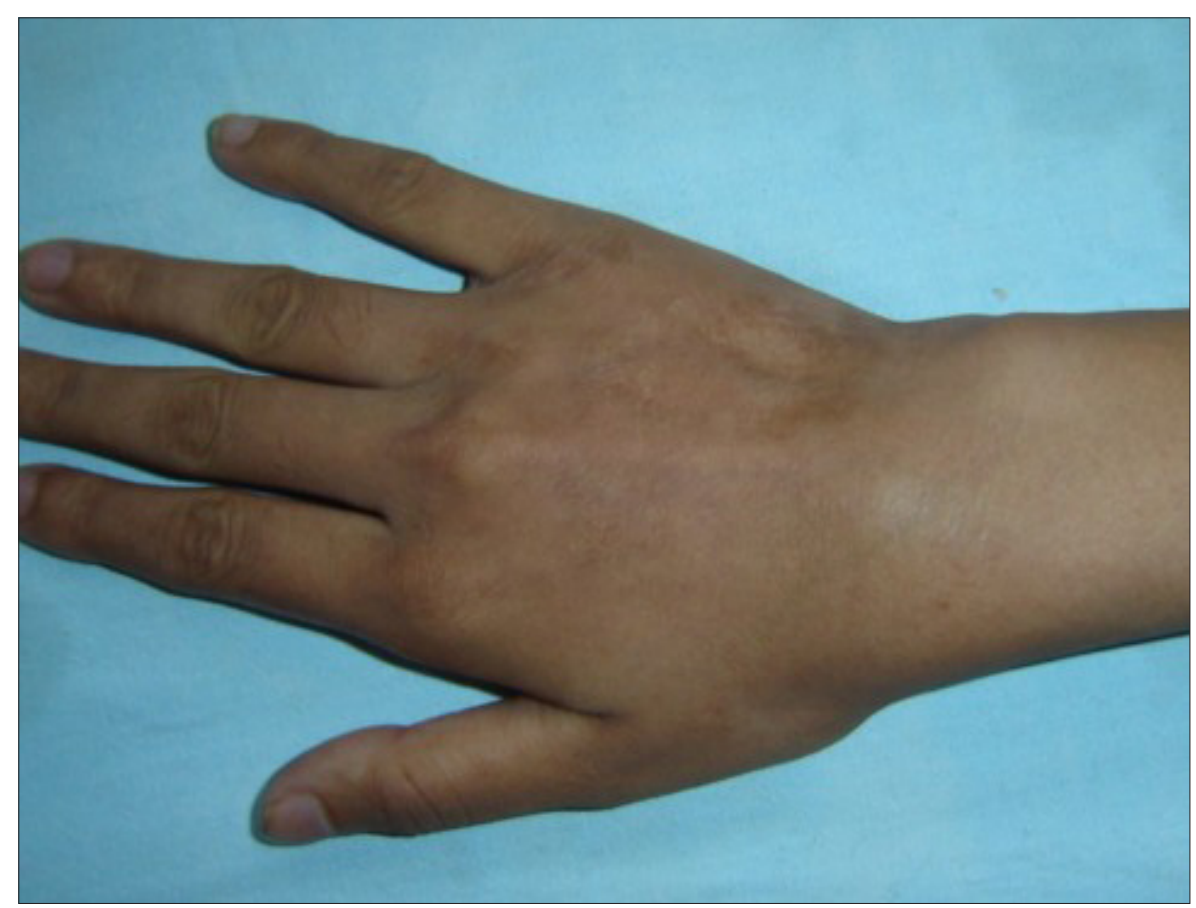

Figure 3: clinical photograph of a patient with chronic synovitis wrist.

Assessment: We tried to correlate our arthroscopic findings with the histopathological diagnosis. Absence of pannus and atrophic villi like architecture was strongly associated (66\% 8 out of 12) with visibility of granulomas on histopathology. Both of the two patients whose synovium revealed rice like bodies showed features suggestive of granulomatous inflammation on histopathology. Overall, 8 out of the 34 patients were diagnosed as having tuberculosis on histopathological grounds [Figure 4,5]. Ziel Nielson staining failed to reveal acid fast bacilli in any of the case. A single patient developed growth of Mycobacterium tuberculosis on culture in LJ medium. 


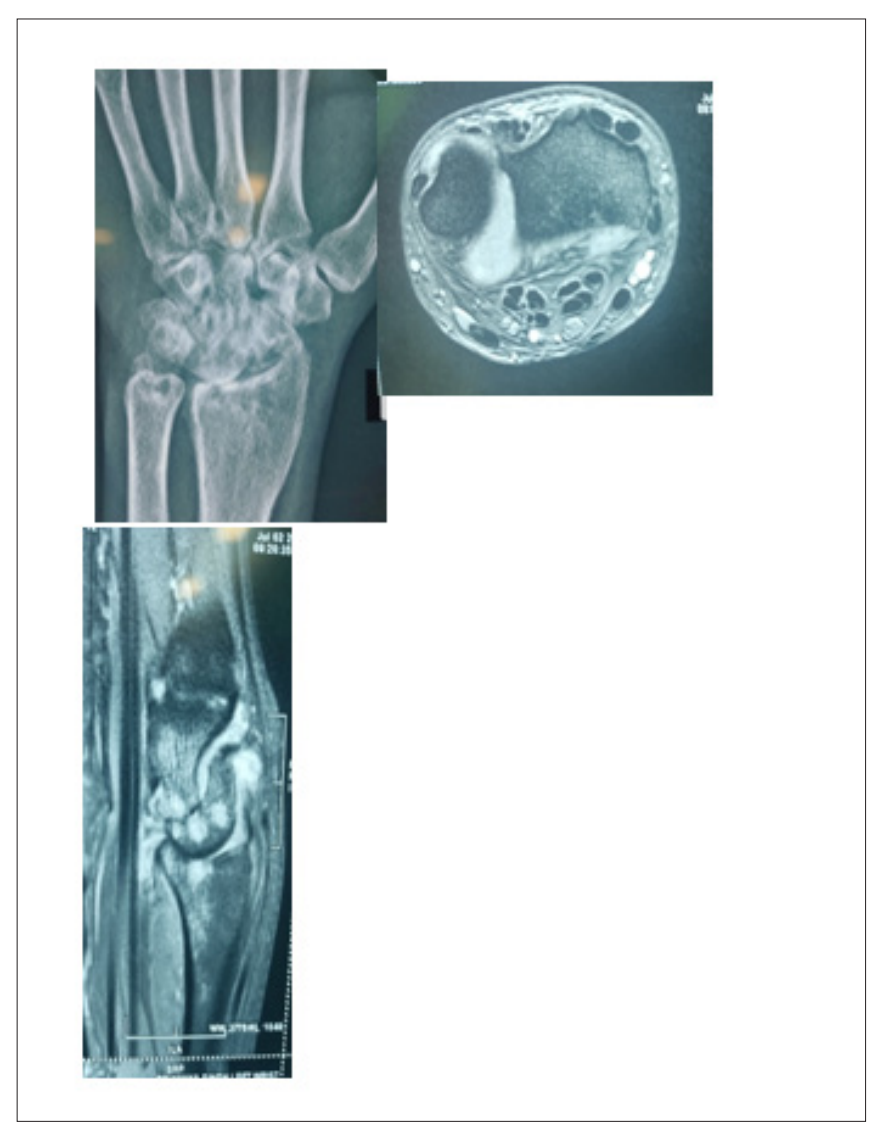

Figure 4: X ray \& MRI images of a typical patient presenting.

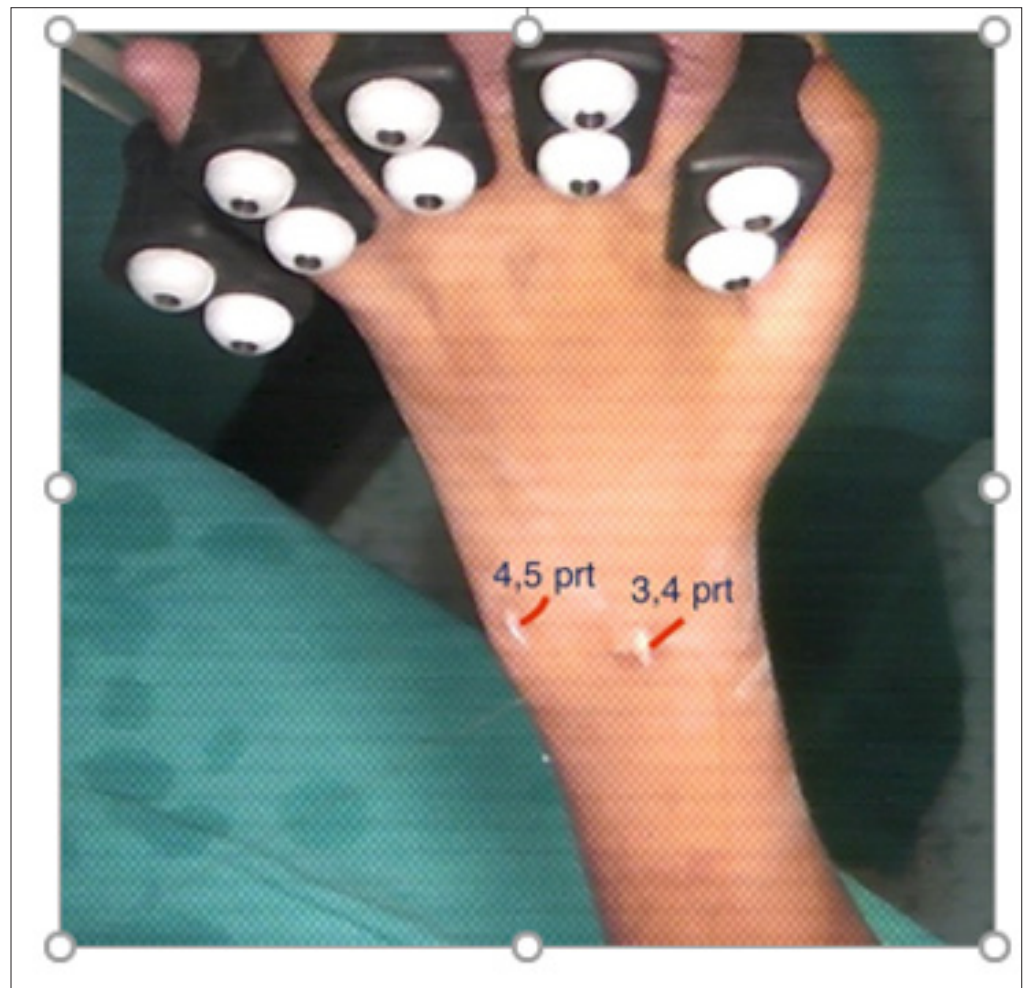

Figure 5: Standard Arthroscopy portal for performing synovectomy. 


\section{Conclusion}

Tuberculosis must be kept as a differential diagnosis in cases presenting as chronic synovitis of wrist especially in tuberculosis endemic zones of southeast Asia. An early arthroscopy and arthroscopic synovial biopsy must be considered in these cases in order to establish a diagnosis. Arthroscopic examination can serve as a good predictor of etiology, but histopathology and culture remain the gold standard.

\section{Conflict of Interest}

None.

\section{Acknowledgements}

None.

\section{References}

1. M J Park, J H Ahn, J S Kang (2017) Arthroscopic synovectomy of wrist in rheumatoid arthritis Hand Clin 33(4): 779-785.

2. Bush D C, Schneider H (1984) Tuberculosis of hand and wrist. J hand Surg (Am) 9(3): 391-398.

3. Nechi porenko V P, Kotskovich I M (1973) formation of rice like bodies. Khin Khir 10: 78-80.

4. Regnard P J, Bassy P, Isselin J (1996) Two cases of thrombosis of the palmar digital vein. J Hand surg (Br) 21(3): 351-354. 